\title{
Bail-out use of Wiggle wire for stuck wire in dissection lumen under optical frequency domain imaging
}

\author{
Jun Shiraishi, Nariko Ikemura, Masayuki Hyogo, Takahisa Sawada \\ Department of Cardiology, Kyoto First Red Cross Hospital, Honmachi, Kyoto, Japan
}

An 80-year-old woman with cerebral infarction complained of chest pain at rest. Coronary angiography (CAG) revealed severe stenosis in the proximal-mid right coronary artery (RCA) (Fig. 1A). The RCA lesion was then dilated with a cutting balloon (2.75/10 mm) at $12 \mathrm{~atm}$ (Fig. 1B). After predilation, optical frequency domain imaging (OFDI) was attempted; however, we could not cross the OFDI catheter through the lesion. After rechecking the CAG findings in detail, we recognized the wire was running outside the RCA curve (Fig. 1C, D). Several attempts were made to advance another guidewire along the inner side of the RCA curve; however, the second guidewire tended to pass through the same route as the first guidewire. We then managed to advance a crooked guidewire (Wiggle, Abbott Vascular) with a double-lumen catheter backup into the distal RCA, and ascertained the Wiggle wire located at the lesser-curvature side on fluoroscopy (Fig. 1E, F). Subsequent OFDI through the Wiggle wire confirmed the Wiggle wire in the true lumen and the first wire in the dissection lumen (Fig. 1G-I). In addition, there was a calcified flap (white arrow, Fig. 1I) at the proximal entry of the dissection lumen, which might interfere with the OFDI catheter crossing through the first wire. A drug-eluting stent (3.0/ $/ 40 \mathrm{~mm}$ ) was implanted through the Wiggle wire without difficulty (Fig. 1J). After postdilations, repeat OFDI depicted full expansion of the stent despite residual dissection lumen (Fig. 1K), and final CAG showed an acceptable result (Fig. 1L).

Conflict of interest: None declared

Address for correspondence: Jun Shiraishi, MD, Department of Cardiology, Kyoto First Red Cross Hospital, Honmachi, Higashiyama-ku, Kyoto 605-0981, Japan, tel: +81-75-561-1121, fax: +81-75-561-6308, e-mail: risa11221998@yahoo.co.jp Received: 7.08.2020 


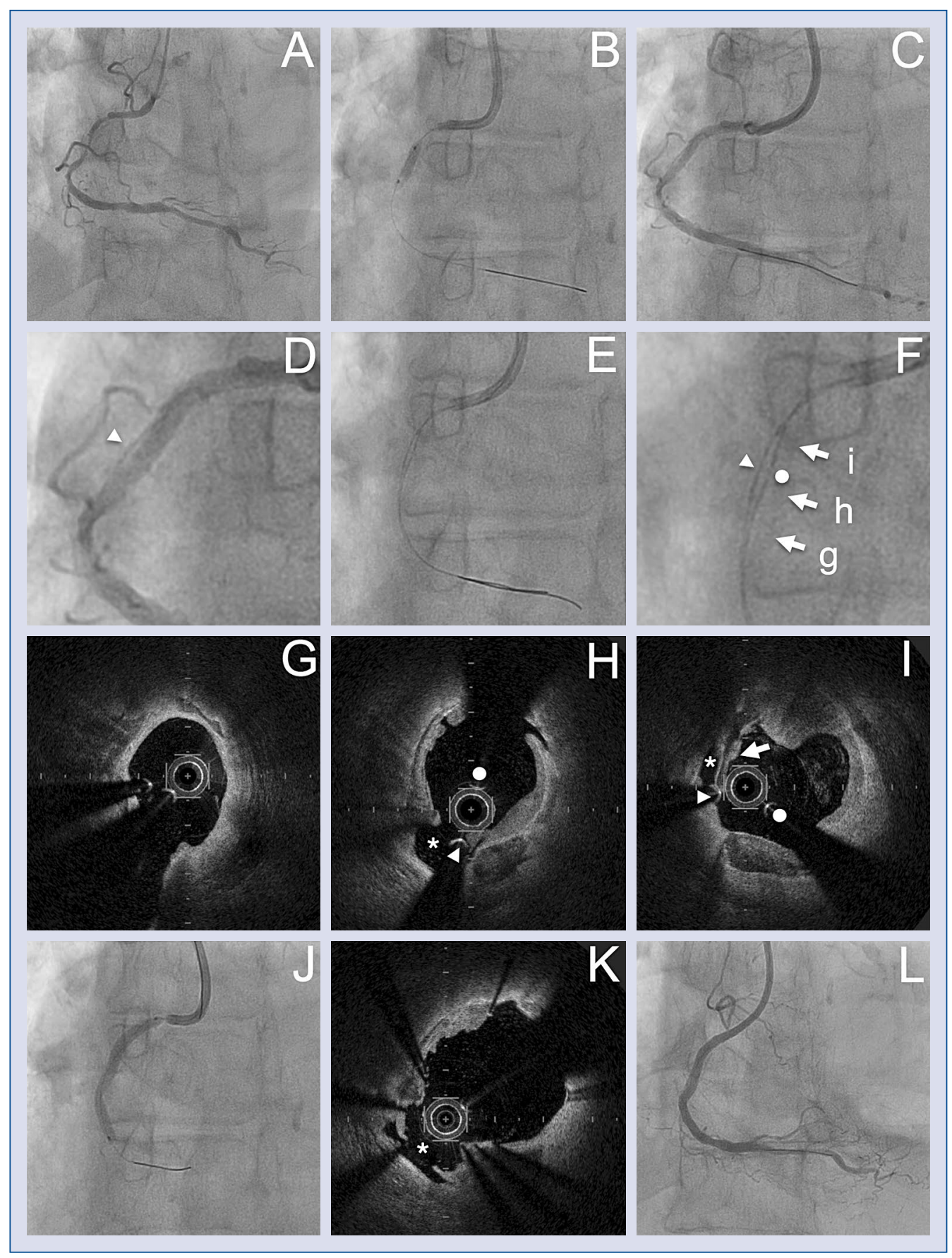

Figure 1. A. Coronary angiography (CAG; left anterior oblique view) showed a significant stenosis in the proximal-mid right coronary artery (RCA); B. Predilation with a cutting balloon; C, D (magnified image). CAG after predilations depicted the first wire (white arrowhead) located at the outer side of the RCA curve; E, F (magnified image). The Wiggle wire (white circle) positioned at the inner side compared with the first wire (white arrowhead) on fluoroscopy. Optical frequency domain imaging (OFDI) images after crossing the Wiggle wire (panels G-I corresponding to arrows in panel F) confirmed the Wiggle wire (white circle) and the first wire (white arrowhead) located in the true lumen and in the dissection lumen (white asterisk), respectively; G. Bifurcation of the right ventricular branch; $\mathbf{H}$. Mid part of the dissection lumen; I. Calcified flap (white arrow) at proximal entry site of the dissection lumen; J. Implantation of a drug-eluting stent; K. Optimal stent expansion/apposition and residual dissection lumen (white asterisk) on final OFDI; L. Final CAG. 